\title{
Respiratory motion correction for enhanced quantification of hepatic lesions in simultaneous PET and DCE-MR imaging
}

RECEIVED

20 July 2020

REVISED

16 March 2021

ACCEPTED FOR PUBLICATION

6 April 2021

PUBLISHED

27 April 2021

Original content from thi work may be used under the terms of the Creative

Commons Attribution 4.0 licence.

Any further distribution of this work must maintain

attribution to the author(s) and the title of the work, journal citation and DOI.

\author{
Matteo Ippoliti ${ }^{1}$ (10) , Mathias Lukas ${ }^{1,2,3}$ (D), Winfried Brenner ${ }^{2}$ (10), Imke Schatka ${ }^{2}$ (D) Christian Furth $^{2}$ (i), \\ Tobias Schaeffter ${ }^{4,5,6}$ (D), Marcus R Makowski ${ }^{1,7, *}$ (i) and Christoph Kolbitsch ${ }^{4, *}$ (iD) \\ 1 Department of Radiology, Charité Universitätsmedizin Berlin, Berlin, Germany \\ 2 Department of Nuclear Medicine, Charité Universitätsmedizin Berlin, Berlin, Germany \\ 3 Siemens Healthcare GmbH, Berlin, Germany \\ 4 Physikalisch-Technische Bundesanstalt (PTB), Braunschweig and Berlin, Germany \\ Technische Universität Berlin, Berlin, Germany \\ King's College London, London, United Kingdom \\ Klinikum rechts der Isar der TU München, Munich, Germany \\ * Contributed equally. \\ E-mail: matteo.ippoliti@charite.de
}

Abstract

Simultaneous positron-emission tomography (PET)-magnetic resonance (MR) imaging is a hybrid technique in oncological hepatic imaging combining soft-tissue and functional contrast of dynamic contrast enhanced MR (DCE-MR) with metabolic information from PET. In this context, respiratory motion represents a major challenge by introducing blurring, artifacts and misregistration in the liver. In this work, we propose a free-breathing $3 \mathrm{D}$ non-rigid respiratory motion correction framework for simultaneously acquired DCE-MR and PET data, which makes use of higher spatial resolution MR data to derive motion information used directly during image reconstruction to minimize image blurring and motion artifacts. The main aim was to increase contrast of hepatic metastases to improve their detection and characterization. DCE-MR data were acquired at 3T through a golden radial phase encoding scheme, enabling derivation of motion fields. These were used in the motion compensated image reconstruction of DCE-MR time-series ( 48 time-points, 6 s temporal resolution, $1.5 \mathrm{~mm}$ isotropic spatial resolution) and 3D PET activity map, which was subsequently interpolated to the DCE-MR resolution. The extended Tofts model was fitted to DCE-MR data, obtaining functional parametric maps related to perfusion such as the endothelial permeability $\left(K_{t}\right)$. Fifty-seven hepatic metastases were identified and analyzed. Quantitative evaluations of motion correction in PET images demonstrated average percentage increases of $16 \% \pm 5 \%$ (mean \pm SD) in Contrast $(C), 18 \% \pm 6 \%$ in $\mathrm{SUV}_{\text {mean }}$ and $14 \% \pm 2 \%$ in $\mathrm{SUV}_{\text {max }}$, while DCE-MR and $K_{t}$ scored contrast-to-noise-ratio increases of $64 \% \pm 3 \%$ and $90 \% \pm 6 \%$, respectively. Motion-corrected data visually showed improved image contrast of hepatic metastases and effectively reduced blurring and motion artefacts. Scatter plots of $S U V_{\text {mean }}$ versus $K_{t}$ suggested that the proposed framework improved differentiation of $K_{t}$ measurements. The presented motion correction framework for simultaneously acquired PETDCE-MR data provides accurately aligned images with increased contrast of hepatic lesions allowing for improved detection and characterization.

\section{Introduction}

Positron-emission tomography (PET) and magnetic resonance (MR) are widely used medical imaging techniques. Simultaneous PET-MR acquisitions are a powerful tool for diagnostic assessment of tumors in oncology (Antoch and Bockisch 2008), combining the variety of complementary functional and morphological image contrasts available from the two modalities. Dynamic contrast-enhanced MR (DCE-MR) imaging is one of the most widely used MR protocols for identifying and characterizing metastases (Choyke et al 2003). It is 
capable of providing both tissue structural information in the form of T1-weighted images and functional information related to perfusion and vascularity in the form of parametric maps obtained using pharmacokinetic modeling of the contrast agent. Functional imaging of hepatic perfusion parameters is especially useful in the detection and characterization of hepatic malignancies and depending on their type and size it can aid in treatment selection and for follow-up investigations (Ronot et al 2016).

PET imaging on the other hand, provides information on metabolic processes in the tissues in exam. The introduction of hybrid scanners capable of performing simultaneous PET-MR imaging has shown to have improved anatomical localization and characterization of hepatic metastases in comparison with conventional imaging (Nasoodi et al 2014), offering a higher diagnostic accuracy in lesion detection (Beiderwellen et al 2015). Combining DCE-MR images and PET derived parameters was demonstrated to provide a high accuracy in comparison to the histopathological grading of hepatocellular carcinoma in a translational rat model (Kaissis et al 2020). Furthermore, a significant level of negative correlation has been observed between the standardized uptake value (SUV) in PET images and parametric maps of perfusion derived from DCE-MR, indicating that hepatocellular carcinomas in their advanced stage and showing signs of high glucose metabolism, generally also show lower perfusion (Ahn et al 2013, Hectors et al 2018). These results suggest that for these specific tumors, behavior could be differentiated through the joint use of PET metabolic maps and functional DCE-MR information regarding perfusion and vascularity, and could in principle be applied to other types of malignancies that show characteristic metabolic-perfusion behaviors.

One of the main challenges for PET-MR imaging in the abdomen is respiratory motion. It results in intraimage artefacts (i.e. blurring and motion artefacts in MR and PET images), inter-image artefacts (i.e. misalignment between DCE time frames) and inter-modality artefacts (i.e. misalignment between MR and PET images if both use different strategies to correct for respiratory motion). These types of artefacts impair the diagnostic quality and power of simultaneous PET-MR acquisitions and the inter-modality artefacts make a voxel-wise analysis of simultaneously acquired PET and parametric DCE-MRI maps challenging.

To address the problem of respiratory motion, advanced and integrated motion correction methods for simultaneous PET-MR imaging based on deriving motion information from higher spatial resolution MR data (McClelland et al 2013, Fürst et al 2015), have been proposed for a variety of applications (Chun et al 2012, King et al 2012, Würslin et al 2013, Manber et al 2015, Fuin et al 2018, Catalano et al 2018). For example, MR-tagging is an approach that creates temporary labels in the images that are then used for tracking the moving anatomy (Chun et al 2012). Alternatively, motion fields (MF) can be derived from registering 2-dimensional multi-slice (Würslin et al 2013, Manber et al 2015) or 3-dimensional (King et al 2012) abdominal MR images that have been acquired over multiple breathing cycles. Recently, Fuin et al (Catalano et al 2018, Fuin et al 2018) introduced a concurrent respiratory motion correction framework for simultaneously acquired DCE-MR and PET that employs an MR radial stack of stars protocol reconstructed through compressed sensing. The main challenge of currently used techniques is the low slice resolution, which could be a limiting factor in the detection of small focal metastases. In addition, to the authors' knowledge, the combination of quantitative DCE maps with PETderived diagnostic parameters, has not been evaluated for dual PET-MR 3D non-rigid respiratory motion correction.

In this work we propose a 3D non-rigid motion correction approach (Ippoliti et al 2019) for simultaneously acquired abdominal PET-DCE-MR data. MF are obtained from diagnostic DCE-MR data and utilized to minimize respiratory motion artefacts in MR and PET data. As both MR and PET utilize the same motion information, inter-modality motion artefacts are also minimized yielding perfectly aligned $\mathrm{PET}$ and quantitative parametric DCE-MR maps. The 3D DCE-MR was acquired with an isotropic spatial resolution of $1.5 \mathrm{~mm}$, ensuring also small lesions could be detected. The effect of motion correction on the combination of quantitative DCE-MR and PET was evaluated by assessing average endothelial permeability and SUV values in a range of lesions.

\section{Methods}

\subsection{Data acquisition}

PET and MR were acquired simultaneously on a 3T Biograph mMR scanner (VE11P, Siemens Healthcare, Erlangen, Germany) for $5 \mathrm{~min}$. The MR sequence is based on a 3D golden-radial phase encoding (GRPE) scheme with Cartesian sampling along the foot-head direction (Buerger et al 2013), with the following characteristic parameters: $\mathrm{TR} / \mathrm{TE}=3.3 \mathrm{~ms} / 1.36 \mathrm{~ms}, \mathrm{FOV}=288 \times(288-345) \times(288-345) \mathrm{mm}^{3}$, flip angle $=12^{\circ}$, partial Fourier factor $=5 / 8$, GRPE lines $=640$, spatial resolution $=1.5 \times 1.5 \times 1.5 \mathrm{~mm}^{3}$. PET data were acquired in listmode immediately after a 2-point Dixon breath-hold sequence to obtain an MR-based attenuation map. This prospective study included 7 patients ( 6 males, $56 \pm 8$ years, $88 \pm 11 \mathrm{~kg}$ ) (mean $\pm \mathrm{SD}$ ) enrolled for suspected or known presence of hepatic metastases, all injected with $169 \pm 14 \mathrm{MBq}$ of ${ }^{68} \mathrm{Ga}$-Dotatoc targeting 
(a)
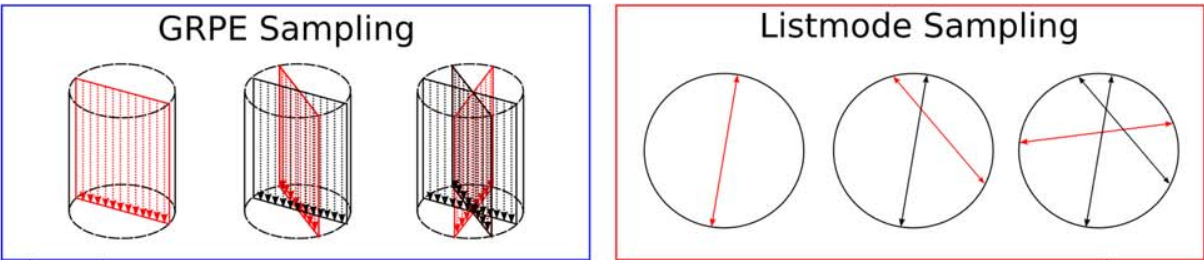

(b)
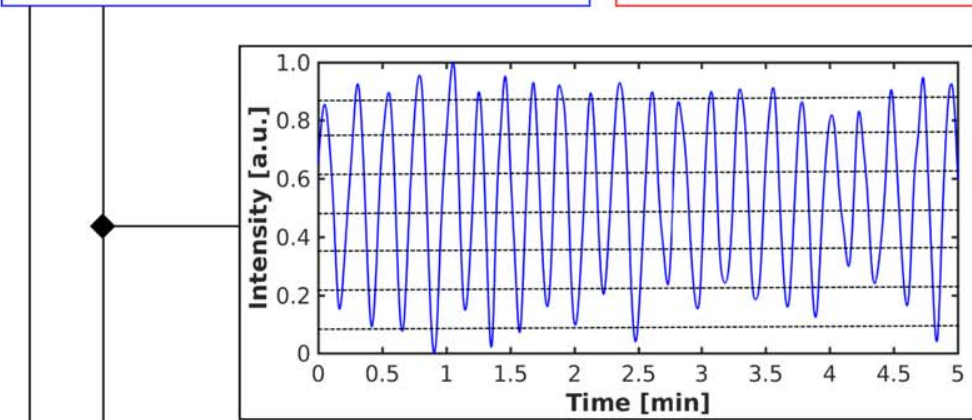

$\downarrow$

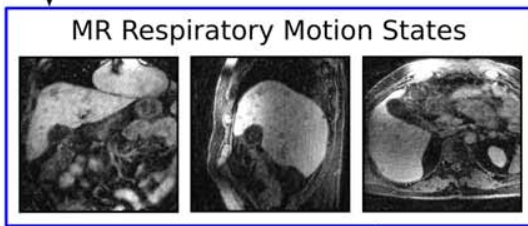

(c)
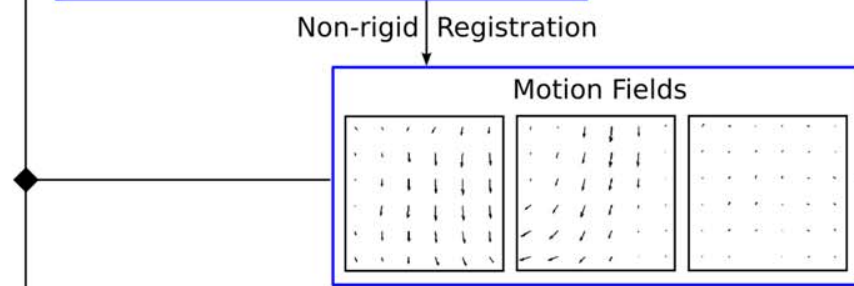

(d)
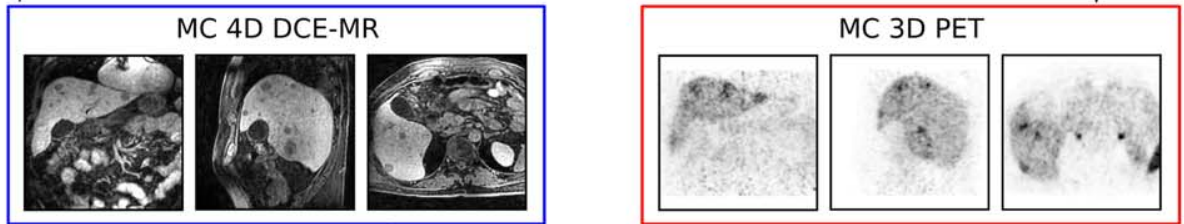

Pharmacokinetic Modelling

(e)

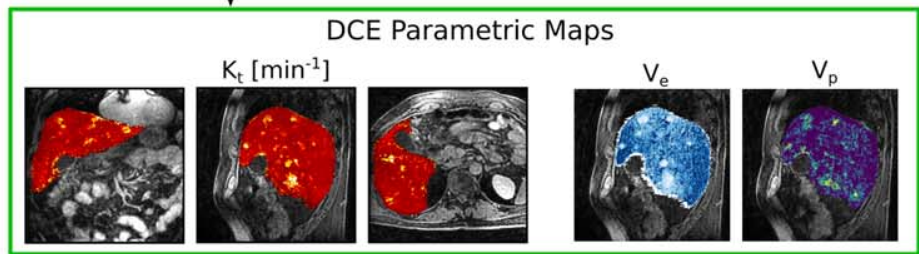

Figure 1. (a) Listmode and GRPE data are acquired simultaneously during free breathing. (b) A respiratory self-navigator is used to divide all MR and PET raw data in 8 respiratory motion states. (c) The MR motion states are reconstructed and registered providing 3D non-rigid motion fields (MF), (d) for motion-corrected image reconstruction of MR and PET data, to yield a 4D DCE-MR timeseries and 3D PET image. (e) Parametric maps of the liver (overlay in color) are obtained from the DCE-MRI (greyscale) using a pharmacokinetic model.

tumoral somatostatin receptors. The PET and MR acquisition started $106 \pm 12$ min post-injection. The MR contrast agent employed was Gadoxeate disodium $\left(0.01 \mathrm{mmol} \mathrm{kg}^{-1}\right)$, which is a hepatospecific contrast agent with memory defect, injected approximately $1 \mathrm{~min}$ after the start of the acquisition.

\subsection{Respiratory motion characterization and MF derivation}

Figure 1 gives an overview of the proposed method. For GRPE a 1D projection through k-space center was repeatedly acquired, which were used to obtain a respiratory self-navigator (Prieto et al 2010). Based on this selfnavigator, the DCE-MR data was split into 8 respiratory motion states and 3D motion-resolved images were 
reconstructed (Cruz et al 2016). The MF were then derived from non-rigidly registering (Rueckert et al 1999) the motion states to a reference (e.g. end-expiration).

\subsection{DCE-MR and PET motion-corrected image reconstruction}

Motion corrected image reconstruction (MCIR) of the DCE-MR time-series was carried out through nonCartesian iterative kt-SENSE (Tsao et al 2003, Hansen et al 2006). Each dynamic phase was split into the different respiratory motion states and then corrected using the MF determined above, directly during image reconstruction. This could be achieved by extracting only the $\mathrm{k}$-space data relative to the motion state being processed and applying the respective MF transformation directly through the encoding operator, together with the fast Fourier transform and the coil sensitivity maps of each receiver employed. This yielded a dynamic dataset where each phase was corrected to the same respiratory motion state.

PET listmode data were binned into sinograms of the 8 different respiratory motion states identified using the MR-based self-navigator. An attenuation correction (AC) map for each subject, was derived from a separate MR scan acquired during an exhale-breathhold. During PET-MCIR, the AC map was transformed together with the listmode data during an iterative ordered subset expectation maximization approach, using the MF derived from MR data (Qiao et al 2006, Dey and King 2009, Kolbitsch et al 2018). In this way the AC map was then transformed during MCIR to match the emission data in the different motion states. Additional registration between the MCIR MR and the AC map was therefore not carried out. Random and scatter correction was applied during image reconstruction (Tsoumpas et al 2004, Polycarpou et al 2010). The final PET images were obtained through an iterative algorithm based on 3D ordered subsets expectation maximization (Thielemans et al 2012) and had the following characteristic parameters: spatial resolution $=2.1 \times 2.1 \times 2.0 \mathrm{~mm}^{3}$, matrix size $=344 \times 344 \times 127,3$ iterations with 23 subsets, post-filtering $3 \mathrm{D}$ Gaussian kernel $=4.0 \times 4.0 \times 4.0$ $\mathrm{mm}^{3}$.

\subsection{Quantitative evaluation of PET-MR motion correction}

Signal intensities in DCE-MR series were converted to $\mathrm{mM}$ concentration of contrast agent using a reference tissue method (Medved et al 2004, DCE MRI Technical Committee 2012). The extended Tofts pharmacokinetic model (Tofts and Kermode 1991, Tofts et al 1999) was then solved extracting three functional parametric maps of interest: $V_{p}$ and $V_{e}$ which represent the fractional volume of contrast agent in blood plasma and in the tissue extravascular extracellular compartment respectively and $K_{t}$ the endothelial permeability. The model behaves according to the following:

$$
C_{\text {liver }}(t)=V_{p} C_{p}(t)+K_{t} e^{-\frac{K_{t}}{V_{e}} \cdot t} * C_{p}(t-\tau),
$$

where $C_{\text {liver }}(t)$ and $C_{p}(t)$ are the concentration of contrast agent sampled in liver tissue and in blood plasma from the hepatic artery respectively, $\tau$ is the time gap between $C_{p}(t)$ and the actual observed tissue enhancement in a given voxel of interest and ${ }^{*}$ denotes the convolution operator.

In order to verify the effects of the proposed PET-MR respiratory motion correction framework, nonmotion-corrected datasets were produced alongside motion-corrected datasets (MC) of all available data (i.e. DCE time-series, SUV, $K_{t}, V_{e}, V_{p}$ maps) for comparison. To quantitatively evaluate the effects of motion correction within each modality, contrast-to-noise-ratio (CNR) analyses in DCE-MR (Ippoliti et al 2019) together with Contrast (C) (Fürst et al 2015), $S_{U V}$ mean and $S_{U V} V_{\text {max }}$ analyses (Kinahan and Karp 1994) in PET were carried out over 57 hepatic lesions identified from all patients. Specifically, the CNR was determined according to the following relation:

$$
\mathrm{CNR}=\frac{I_{\text {metastasis }}-I_{\text {surround }}}{\sigma_{\text {surround }}}
$$

where $I_{\text {metastasis }}$ and $I_{\text {surround }}$ represent the mean signal registered inside a region of interest (ROI) containing the lesion and a ROI of comparable size which directly surrounds it, respectively. $\sigma_{\text {surround }}$ is the standard deviation of this latter ROI. As for the $C$ measured in PET, the following equation holds:

$$
C=\frac{A_{\text {metastasis }}-A_{\text {background }}}{A_{\text {background }}},
$$

where $A_{\text {metastasis }}$ represents the mean activity in lesion ROI and $A_{\text {background }}$ is the mean activity in liver background tissue, which is taken from a rectangular cuboidal ROI that does not contain focal tracer uptake or edge voxels. The background ROI was therefore not taken from tissue surrounding the lesion as was done for the DCE-MR part.

ROIs in PET were obtained by manually drawing a spherical mask encompassing the lesion in both NMC and MC datasets. The starting size of the spherical mask was identical in the two datasets and was simply placed around the lesion in order to fully encapsulate it. Then, the top $10 \%$ of SUV scoring voxels $\left(\mathrm{ROI}_{\mathrm{PET}}^{10 \%}\right)$ was selected 

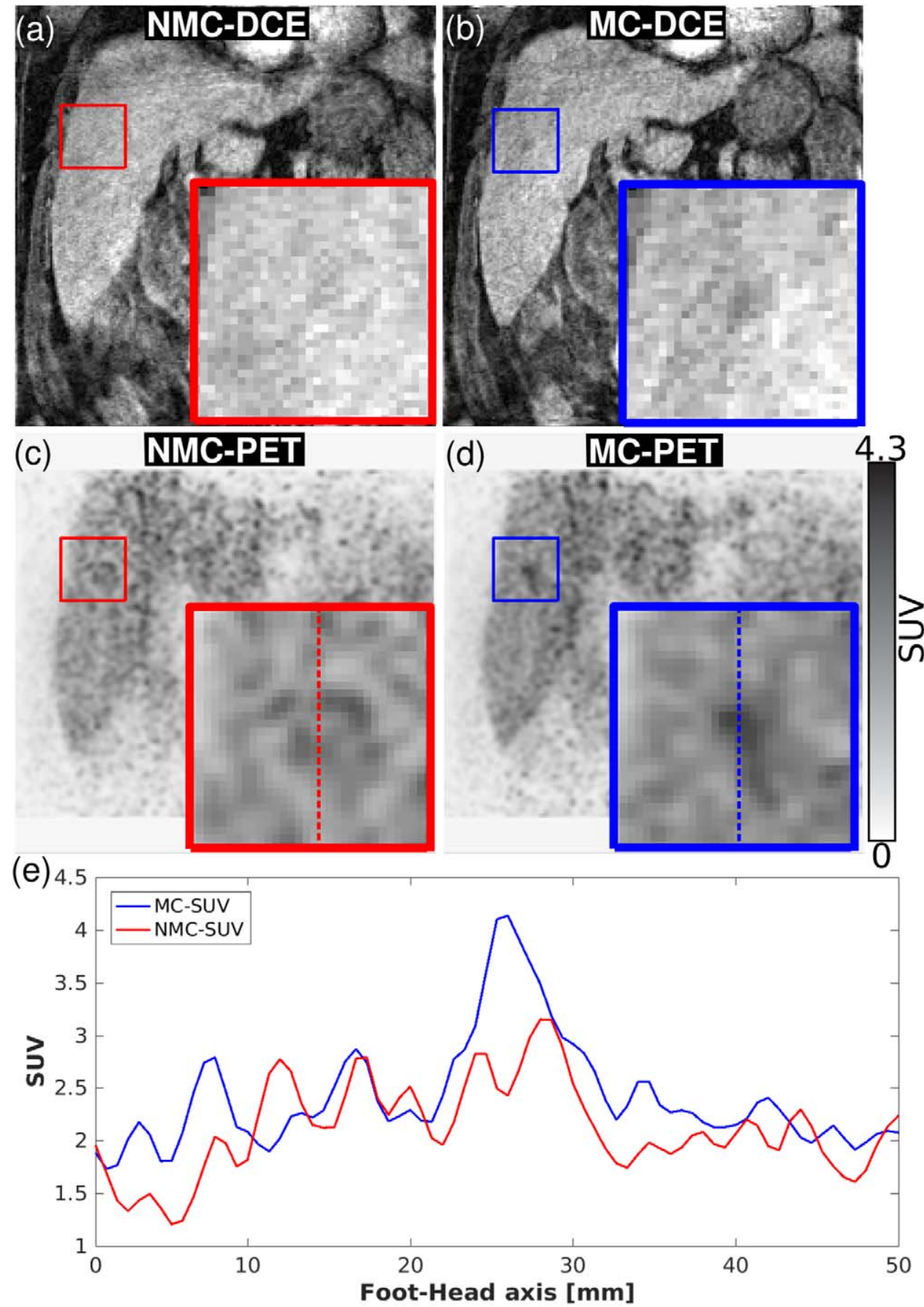

Figure 2. Comparison of small lesion from Patient 1 between (a) NMC-DCE, (b) MC-DCE, (c) NMC-PET, (d) MC-PET images and (e) plot of SUV line profile passing through the lesion's center. Respiratory motion correction reduces blurring and increases lesion contrast with respect to surrounding healthy tissue.

by thresholding. In this manner, the size of the ROI varied between $38 \mathrm{~mm}^{3}$ and $1073 \mathrm{~mm}^{3}$ depending on the different lesions. DCE-MR masks (ROI $\mathrm{DCE}_{\text {) }}$ were also drawn manually, according to lesion boundaries in the latest DCE image of each patient's time-series and were used on the DCE image and on the $K_{t}, V_{e}$ and $V_{p}$ functional parametric maps, to carry out CNR analyses. $\mathrm{ROI}_{\mathrm{DCE}}$ masks were drawn separately in the MC and NMC datasets and sizes were kept comparable.

In order to investigate the effect of motion correction on the dual-modality characterization of lesions, we assessed the distribution of $\mathrm{SUV}_{\text {mean }}$ and average endothelial permeability $\left(\overline{K_{t}}\right)$ in 31 metastases from a single patient suffering from neuroendocrine tumors. The comparison was carried out between motion-corrected data and uncorrected data. All values were calculated as the average over a ROI with the same number of voxels for $\mathrm{SUV}_{\text {mean }}$ and $K_{t}$. Statistical significance in the difference of mean values of all the above mentioned quantities for both DCE-MR and PET, was tested for with paired sample t-tests, after checking the normality distribution 


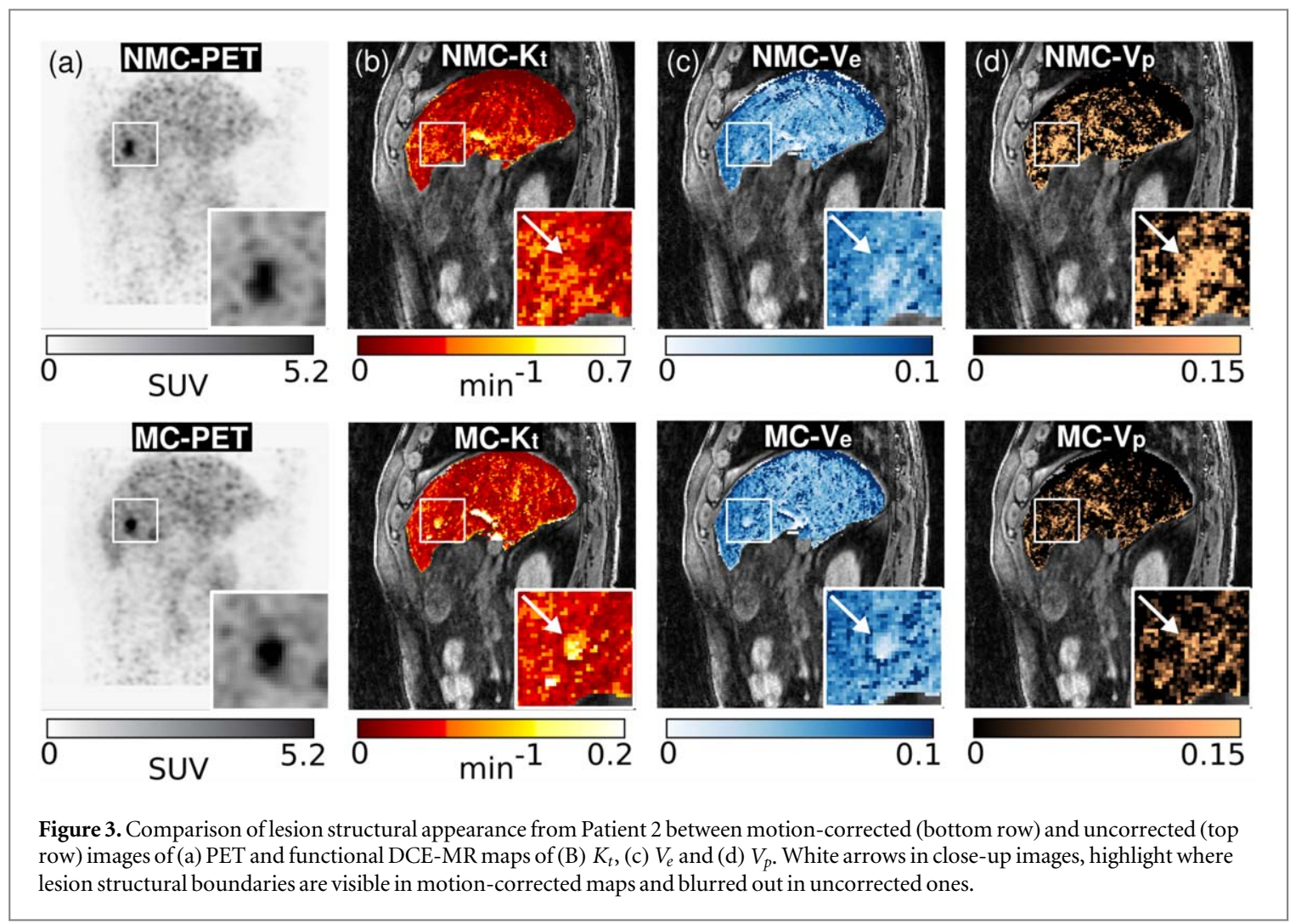

Table 1. CNR evaluation in DCE-MR together with $C$ and SUV evaluations in PET.

\begin{tabular}{lccc}
\hline Parameter & $\mathrm{MC}^{\mathrm{a}}$ & $\mathrm{NMC}^{\mathrm{a}}$ & $\mathrm{MC}-\mathrm{NMC}(\%)^{\mathrm{b}}$ \\
\hline$\overline{\mathrm{CNR}}_{\mathrm{DCE}}$ & $1.46 \pm 0.88$ & $0.88 \pm 0.59$ & $65 \pm 4$ \\
$\overline{\mathrm{CNR}}_{K t}$ & $1.11 \pm 0.81$ & $0.57 \pm 0.50$ & $96 \pm 8$ \\
$\overline{\mathrm{CNR}}_{V e}$ & $0.35 \pm 0.27$ & $0.29 \pm 0.27$ & $21 \pm 14$ \\
$\overline{\mathrm{CNR}}_{V p}$ & $0.25 \pm 0.24$ & $0.20 \pm 0.16$ & $78 \pm 14$ \\
$\overline{\mathrm{C}}$ & $1.94 \pm 1.26$ & $1.67 \pm 1.18$ & $16 \pm 5$ \\
$\overline{\mathrm{SUV}}_{\text {mean }}$ & $6.2 \pm 3.1$ & $5.2 \pm 3.0$ & $19 \pm 6$ \\
$\overline{\mathrm{SUV}}_{\text {MAX }}$ & $7.2 \pm 3.7$ & $6.3 \pm 3.4$ & $14 \pm 3$ \\
\hline $\bar{S}$ & & &
\end{tabular}

${ }^{8}$ Values are mean \pm SD.

${ }^{9}$ Values are average percentage variation \pm statistically propagated uncertainty, computed using mean values and SD for MC and NMC data.

assumption was fulfilled by the sampled data, by employing a 1-sample Kolmogorov-Smirnov test. Differences in the $\mathrm{t}$-tests were considered to be statistically significant for $p<0.05$. The image reconstruction algorithms for $\mathrm{MR}$, together with the algorithms for carrying out quantitative evaluation procedures and for resolving the extended Tofts model, were written in MATLAB (R2016b, The MathWorks, Natick, MA) and Python (Python Software Foundation). The PET reconstruction algorithm was implemented in $\mathrm{C}++$ (International Standard ISO/IEC 14882:2017(E)_Programming Language $\mathrm{C}++$ ), but its precompiled functions were also called through Matlab.

\section{Results}

\subsection{Quantitative evaluation of motion correction on DCE-MR and PET data}

Figure 2 shows the DCE and static 3D PET images of Patient 1 with and without motion correction. The small lesion highlighted is almost completely indistinguishable from the background tissue in the NMC-DCE and NMC-PET images, while it is clearly visible in the MC-DCE and MC-PET. Furthermore, the line profile in figure 2(e) shows a percentage difference of $52 \%$ in the measured $S U V_{\text {mean }}$ peak values between MC-PET and NMC-PET images ( $\mathrm{SUV}_{\text {mean }}$ of 4.1 and of 2.7 respectively). The quantitative evaluation of the CNR over the 57 

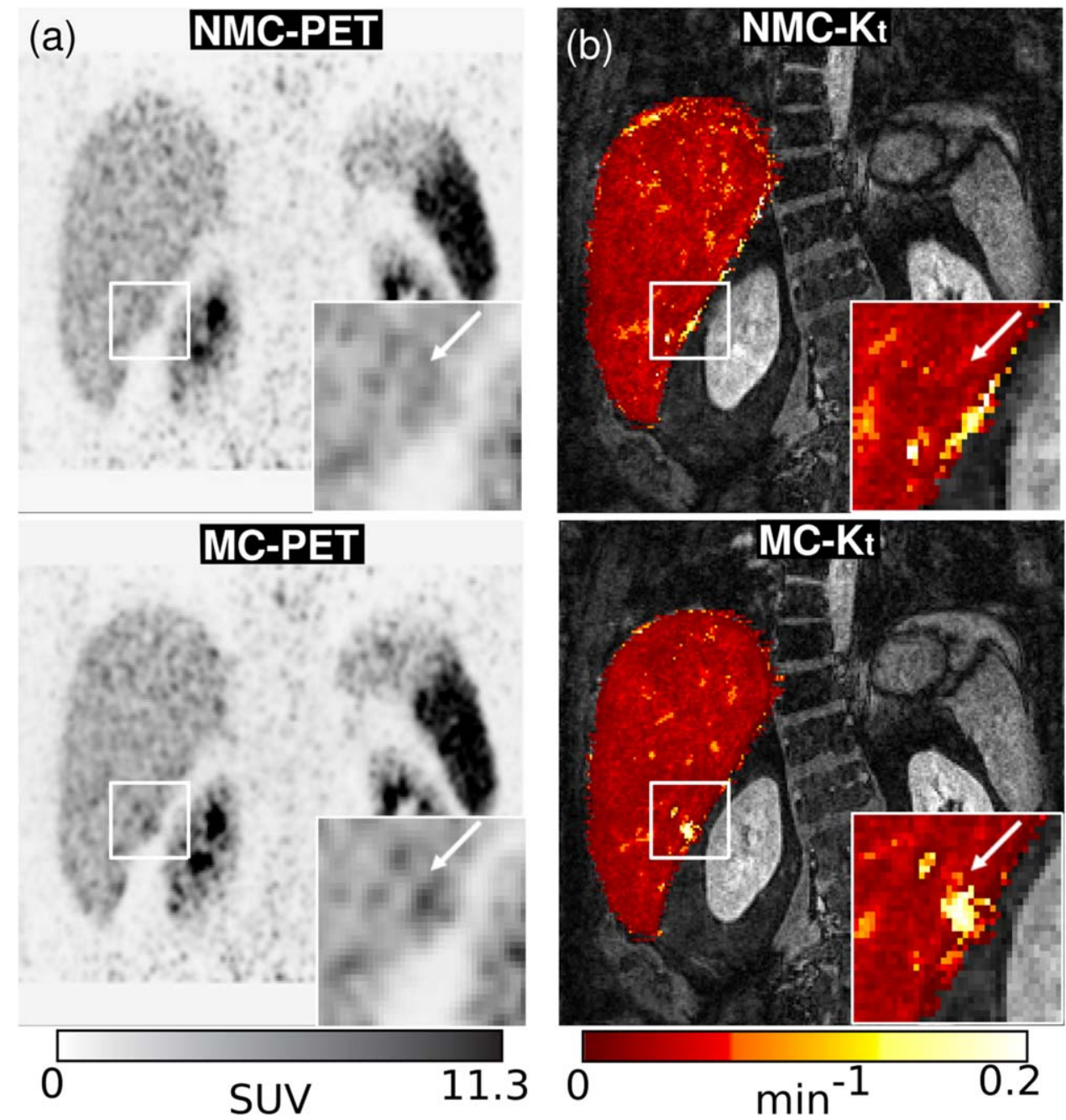

Figure 4. Small hepatic metastasis from Patient 3 reported and compared in motion-corrected (bottom row) and uncorrected (top row) images of (a) PET activity and (b) endothelial permeability $K_{t}$ maps. White arrows indicate lesion location, which is visible in motion-corrected images and vanishes in uncorrected ones.

examined lesions reported in table 1 shows a statistically significant increase in the MC-DCE datasets, scoring a percentage increase $\triangle \mathrm{CN} \mathrm{NCE}_{\mathrm{DCE}}$ of $65 \% \pm 4 \%$. Similarly, in the MC-PET images we also find an increase in contrast that yields a $\Delta \bar{C}=16 \% \pm 5 \%$, in line with increases found in $\operatorname{SUV}_{\max }\left(\Delta S \bar{U} V_{\max }=14 \% \pm 3 \%\right)$ and $\operatorname{SUV}_{\text {mean }}\left(\Delta S \bar{U} V_{\text {mean }}=19 \% \pm 6 \%\right)$. The differences in all mean values evaluated through the paired sample $\mathrm{t}$-tests and reported in table 1 were statistically significant $(p<0.05)$. The maximum motion displacement was calculated for each lesion along the foot-head direction by calculating the maximum of the registered displacement from MF over all motion states for each voxel and then averaging this within each $\mathrm{ROI}_{\mathrm{DCE}}$ mask. The average displacement of all lesions was calculated to be $4.76 \pm 2.16 \mathrm{~mm}$.

\subsection{Analysis of DCE-MR functional parametric maps}

Figure 3 depicts a sagittal orientation of the PET image, $K_{t}$ and $V_{e}$ maps of Patient 2, highlighting a large hepatic lesion. The lesion appears to be more focal in terms of intensity and size in the MC-PET dataset with respect to its NMC-PET twin. From the parametric maps, one can appreciate the strong differences brought by the proposed motion correction framework in terms of lesion size, contrast and boundaries. This effect can also be verified immediately by visually comparing the different parametric maps contained in figures 3 and 4 , which respectively show a coronal and sagittal orientation of Patients 2 and 3 . In figure 4 it can also be seen how all uncorrected images have suffered from blurring over the focal lesion and resulted in its complete disappearance from the PET dataset. On the other hand, the motion-corrected datasets all identify clearly the presence of two separate lesions, which also comply in terms of shape and size across the different images. The above observations are in line with findings regarding the quantitative $\overline{\mathrm{CNR}}$ evaluations over the DCE parametric maps reported in table 1, which show for $K_{t}$ a $\Delta \overline{\mathrm{CNR}}_{K t}=90 \% \pm 6 \%$, for $V_{e}$ a $\Delta \overline{\mathrm{CNR}}_{V e}=19 \% \pm 13 \%$ and for $V_{p}$ a $\Delta \overline{\mathrm{CNR}}_{V p}=76 \% \pm 13 \%$. 



Figure 5. Scatter plots of 31 lesions and 4 healthy tissue ROIs (green circles) depicting (a) uncorrected and (b) motion-corrected landscapes as a function of $\overline{K_{t}}$ and $\mathrm{SUV}_{\text {mean }}$. Contrast uptake curves, pharmacokinetic fit and input function sampled in the hepatic artery $C_{p}$, of two lesions with comparable $S U V_{\text {mean }}$, but with (c) low $K_{t}$ (yellow circle) and (d) high $K_{t}$ (purple circle) respectively.

\subsection{Assessment of motion correction on permeability-PET coupling for hepatic characterization}

The effects of PET-MR motion correction on the characterization of 31 lesions in one patient as a function of $\mathrm{SUV}_{\text {mean }}$ and $\overline{K_{t}}$, is shown in figures 5 (a) and (b). Green circles represent samples of healthy liver parenchyma for reference purpose. The mean permeability values (and SD) measured in healthy tissue are similar for MC- $\overline{K_{t}}$ $\left(0.030 \pm 0.010 \mathrm{~min}^{-1}\right)$ and for NMC- $\overline{K_{t}}$ data $\left(0.034 \pm 0.014 \mathrm{~min}^{-1}\right) . \mathrm{MC}-\mathrm{SUV}_{\text {mean }}$ values on the other hand are $33 \% \pm 3 \%$ higher $(2.23 \pm 0.08)$ compared to the NMC-SUV mean $(1.68 \pm 0.13)$. The mean $\overline{K_{t}}$ and SUV mean, averaged over all lesions, are $96 \% \pm 3 \%$ and $9 \% \pm 3 \%$ higher for motion-corrected data with respect to the uncorrected one, respectively. The DCE-MR contrast uptake curves with the corresponding pharmacokinetic fits and hepatic input functions $C_{p}$ of two lesions with similar high $S U V_{\text {mean }}$ but low (yellow circle) and high (purple circle) $\overline{K_{t}}$ are shown in figure 5(c) (low $K_{t}$ ) and figure 5(d) (high $K_{t}$ ). The fitting of the DCE-MR timeseries was carried out through a non-linear least squares method.

\section{Discussion}

This work demonstrated that non-rigid respiratory motion correction yields high image quality of both quantitative DCE-MR and simultaneously acquired PET data. Contrast and SUV for PET and CNR for DCEMRI was increased by up to $16 \% \pm 5 \%$. Furthermore, this approach ensures that images from both modalities are well aligned, allowing for multi-parametric quantitative assessment of lesions.

For DCE-MR and functional maps, the increase in CNR in the MC images can be explained by reducing motion artefacts in each dynamic image (intra-image artefacts) and by ensuring that the different images are all in the same respiratory motion states and hence well aligned (inter-image artefacts) (figure 3). Furthermore, MC maps are more similar in terms of their value distribution across different patients compared to the NMC maps (see $K_{t}$ figures 3 and 4 ). The percentage increases in $C, S_{U V}$ mean and $S U V_{\max }$ are also significant and all in line with recent findings (Fürst et al 2015, Catalano et al 2018, Fuin et al 2018). In some instances, like in figure 4, where focal lesions of only few millimeters in size are present, the proposed framework has shown features that could not be reproduced without motion correction, in both PET and MR data. The line profile of figure 2(e) demonstrates that the lesion is strongly blurred due to respiratory motion in terms of $\mathrm{SUV}_{\text {mean }}$ values $(52 \%$ percentage difference in measured $\mathrm{NMC}-\mathrm{SUV}_{\text {mean }}$ and $\mathrm{MC}-\mathrm{SUV}_{\text {mean }}$ ) and cannot be distinguished from healthy background tissue without motion correction. In cases like figures 2 and 4, motion correction enabled the 
detection of lesions which would have otherwise been missed or underestimated, as is also confirmed by the measured increase of $C$ and SUV values found for MC-PET images and for the MC- $K_{t}$ maps $(90 \% \pm 6 \%)$.

Recently, a work proposed by Fuin et al (Catalano et al 2018, Fuin et al 2018) has demonstrated the feasibility of performing respiratory motion correction of simultaneously acquired PET-MR data, based on the derivation of MF from radial MR data reconstructed through a compressed sensing approach. Although the method successfully applies motion correction increasing contrast and SUV of lesions in PET, it may not provide sufficient slice resolution to intercept smaller metastases, as is accounted by the highest $\mathrm{SUV}_{\max }$ measurements errors which are primarily found in lesions smaller than $10 \mathrm{~mm}$ in diameter (Catalano et al 2018).

The framework proposed in this paper yields quantitative DCE-MR images which provide functional information about tumors highly complementary to the PET data, all with $1.5 \mathrm{~mm}$ isotropic resolution and without the need for any breath-holds or external devices. Due to the high spatial and temporal resolution of the used DCE-MR acquisition, residual undersampling artefacts are visible as noise-like signal variations in the uptake curves. Nevertheless, due to the incoherence of these artefacts over time, robust parameter estimation was still possible. Furthermore, the comparison between the $\mathrm{SUV}_{\text {mean }}$ and $\overline{K_{t}}$ (figure 5 ) together with the evaluation of the uptake curves of the high and low $K_{t}$ lesions, suggest that MC provides increased sensitivity for both SUV values and functional DCE parameters. The plot in figure 5 shows that the distribution of $K_{t}$ without motion correction is shifted to lower values, leading to an overlap of healthy tissue and low-perfusion lesions. With motion correction, $K_{t}$ values for healthy tissue are clearly separated from lesions. The underestimation of perfusion due to respiratory motion artefacts is also shown in figures 5 (c) and (d) for lesions with low and high $K_{t}$-values. On the one hand, blurring due to respiratory motion leads to an underestimation of contrast agent concentration. On the other hand, respiratory motion artefacts also lead to a worse fit of data and pharmacokinetic model and hence inaccurate parameter estimation. Both sources of error are reduced with the proposed motion correction approach.

One limitation in this study is that we could not verify the accuracy of the motion estimation. To the best of our knowledge there is no phantom available which allows for the acquisition of DCE-MRI and simultaneous PET in the liver during respiratory motion. The MCIR of the DCE-MR has to be carried out for each of the dynamics and requires several hours. This limits the application of the proposed approach in clinical practice with the currently available computer systems. Finally, spatial distortion corrections in MR were not carried out in this work and this could lead to inaccurate motion correction towards the edge of the field of view.

In the present study, partial volume correction has not been applied in the PET image reconstruction process. Especially for the MCIR PET data this could further improve the quantification of the tracer uptake as has been shown by Petibon et al (2014).

The findings concerning the quantitative evaluation of $K_{t}$ in liver metastases reported in this study are in good agreement with other DCE-MR motion correction analyses carried out using DCE-VIBE (Zheng et al 2015). Zheng et al, report average $K_{t}$ values of $0.25 \pm 0.08 \mathrm{~min}^{-1}$, after the application of motion correction through non-rigid registration. Furthermore, an improved estimation of $\mathrm{SUV}_{\text {mean }}$ versus $\overline{K_{t}}$ could in principle aid in oncological abdominal applications such as the characterization of advanced hepatocellular carcinoma where increased SUV- $K_{t}$ sensitivity is required (Ahn et al 2013, Hectors et al 2018). Further clinical studies integrating histopathology would nevertheless be required in order to confirm this assumption.

\section{Conclusion}

The presented free-breathing PET-MR respiratory motion correction framework yields a dynamic DCE-MR time-series and a 3D PET activity map for each patient, with isotropic spatial resolution of $1.5 \mathrm{~mm}$. The framework minimizes motion artifacts and blurring in the images, increasing the overall registration intra- and inter-modality, allowing for the extraction of quantitative information from the MR data and increasing contrast in PET and DCE-MR measurements of lesions. Furthermore, it takes advantage of the high slice resolution together with multiple quantitative biophysical parameters, to provide a comprehensive evaluation tool for hepatic lesions.

\section{Acknowledgments}

The authors gratefully acknowledge funding from the German Research Foundation (INST 335/543-1 FUGGPET/MR, GRK 2260-BIOQIC and DFG-CRC 1340-Matrix in Vision). Mathias Lukas is also employed by Siemens Healthcare GmbH. No other potential conflict of interest relevant to this article was reported. 


\section{Ethical statement}

This prospective study included 7 patients who provided their written informed consent and was prospectively approved by and registered with the local Institutional Review Board (Trial Registration Number: EA4/051/17).

\section{ORCID iDs}

Matteo Ippoliti (i) https://orcid.org/0000-0002-3019-7647

Mathias Lukas @ https://orcid.org/0000-0002-5100-4496

Winfried Brenner (1) https://orcid.org/0000-0003-2478-6004

Imke Schatka (1) https:// orcid.org/0000-0002-2628-6145

Christian Furth (1) https://orcid.org/0000-0001-7302-7886

Tobias Schaeffter (i) https://orcid.org/0000-0003-1310-2631

Marcus R Makowski (i) https://orcid.org/0000-0001-7719-8236

Christoph Kolbitsch ๑ https://orcid.org/0000-0002-4355-8368

\section{References}

Ahn S J, Park M S, Kim K A, Park J Y, Kim I S, Kang W J, Lee S K and Kim M J $2013{ }^{18}$ F-FDG PET metabolic parameters and MRI perfusion and diffusion parameters in hepatocellular carcinoma: a preliminary study PLoS One 8 e71571

Antoch G and Bockisch A 2008 Combined PET/MRI: a new dimension in whole-body oncology imaging? Eur. J. Nucl. Med. Mol. Imaging 36 $113-20$

Beiderwellen K, Geraldo L, Ruhlmann V, Heusch P, Gomez B, Nensa F, Umutlu L and Lauenstein T C 2015 Accuracy of [18F]FDG PET/ MRI for the detection of liver metastases PLoS One 10 e 0137285

Buerger C, Prieto C and Schaeffter T 2013 Highly efficient 3D motion-compensated abdomen MRI from undersampled golden-RPE acquisition MAGMA 26419-29

Catalano O A et al 2018 Comparison of the clinical performance of upper abdominal PET/DCE-MRI with and without concurrent respiratory motion correction (MoCo) Eur. J. Nucl. Med. Mol. Imaging $452147-54$

Choyke P L, Dwyer A J and Knopp M V 2003 Functional tumor imaging with dynamic contrast-enhanced magnetic resonance imaging J. Magn. Reson. Imaging 17 509-20

Chun S Y, Reese T G, Ouyang J, Guerin B, Catana C, Zhu X, Alpert N M and El Fakhri G 2012 MRI-based non-rigid motion correction in simultaneous PET/MRI J. Nucl. Med. 53 1284-91

Cruz G, Atkinson D, Buerger C, Schaeffter T and Prieto C 2016 Accelerated motion corrected three-dimensional abdominal MRI using total variation regularized SENSE reconstruction Magn. Reson. Med. 75 1484-98

DCE MRI Technical Committee 2012 DCE MRI Quantification Profile, Quantitative Imaging Biomarkers Alliance. Version 1.0. Reviewed Draft. QIBA, July 1, 2012. Available from: http://rsna.org/QIBA_aspx

Dey J and King M A 2009 Theoretical and numerical study of MLEM and OSEM reconstruction algorithms for motion correction in emission tomography IEEE Trans. Nucl. Sci. 56 2739-49

Fuin N, Catalano O A, Scipioni M, Canjels L P W, Izquierdo-Garcia D, Pedemonte S and Catana C 2018 Concurrent respiratory motion correction of abdominal PET and DCE-MRI using a compressed sensing approach J. Nucl. Med. 59 1474-9

Fürst S, Grimm R, Hong I, Souvatzoglou M, Casey M E, Schwaiger M, Nekolla S G and Ziegler S I 2015 Motion correction strategies for integrated PET/MR J. Nucl. Med. 56 261-9

Hansen M S, Baltes C, Tsao J, Kozerke S, Pruessmann K P and Eggers H 2006 k-t BLAST reconstruction from non-Cartesian k-t space sampling Magn. Reson. Imaging 55 85-91

Hectors S J, Wagner M, Besa C, Huang W and Taouli B 2018 Multiparametric FDG-PET/MRI of hepatocellular carcinoma: initial experience Contrast Media Mol. Imaging 20185638283

Ippoliti M, Lukas M, Brenner W, Schaeffter T, Makowski M R and Kolbitsch C 2019 3D nonrigid motion correction for quantitative assessment of hepatic lesions in DCE-MRI Magn. Reson. Med. 82 1753-66

Kaissis G A et al 2020 Combined DCE-MRI- and FDG-PET enable histopathological grading prediction in a rat model of hepatocellular carcinoma Eur. J. Radiol. 124108848

Kinahan P E and Karp J S 1994 Figures of merit for comparing reconstruction algorithms with a volume-imaging PET scanner Phys. Med. Biol. 39631-42

King A P, Buerger C, Tsoumpas C, Marsden P K and Schaeffter T 2012 Thoracic respiratory motion estimation from MRI using a statistical model and a 2D image navigator Med. Image Anal. 16252-64

Kolbitsch C, Neji R, Fenchel M, Mallia A, Marsden P and Schaeffter T 2018 Fully integrated 3D high-resolution multicontrast abdominal PET-MR with high scan efficiency Magn. Reson. Med.79900-11

Manber R, Thielemans K, Hutton B F, Barnes A, Ourselin S, Arridge S, O’Meara C, Wan S and Atkinson D 2015 Practical PET respiratory motion correction in clinical PET/MR J. Nucl. Med. 56 890-6

McClelland J R, Hawkes D J, Schaeffter T and King A P 2013 Respiratory motion models: a review Med. Image Anal. 17 19-42

Medved M, Karczmar G, Yang C, Dignam J, Gajewski T F, Kindler H, Vokes E, MacEneany P, Mitchell M T and Stadler W M 2004 Semiquantitative analysis of dynamic contrast enhanced MRI in cancer patients: variability and changes in tumor tissue over time J. Magn. Reson. Imaging $20122-8$

Nasoodi A, Syed R, Afaq A and Bomanji J 2014 Use of PET/MRI for identification and characterisation of liver lesions Clin. Transl. Imaging. 2129-37

Petibon Y, Huang C, Ouyang J, Reese T G, Li Q, Syrkina A, Chen Y - L and El Fakhri G 2014 Relative role of motion and PSF compensation in whole-body oncologic PET-MR imaging Med. Phys. 41042503

Polycarpou I, Thielemans K, Manjeshwar R, Aguiar P, Marsden P K and Tsoumpas C 2010 Comparative evaluations of scatter correction in 3D PET using different scatter-level approximations Ann. Nucl. Med. 25 643-9 
Prieto C, Uribe S, Razavi R, Atkinson D and Schaeffter T 2010 3D undersampled golden-radial phase encoding for DCE-MRA using inherently regularized iterative SENSE Magn. Reson. Imaging 64 514-26

Qiao F, Pan T, Clark J W Jr and Mawlawi O R 2006 A motion-incorporated reconstruction method for gated PET studies Phys. Med. Biol. 51 3769-83

Ronot M, Clift A K, Vilgrain V and Frilling A 2016 Functional imaging in liver tumors J. Hepatol. 65 1017-30

Rueckert D, Sonoda L I, Hayes C, Hill D L G, Leach M O and Hawkes D J 1999 Nonrigid registration using free-form deformations: application to breast MR images IEEE Trans. Med. Imaging 18 712-21

Thielemans K, Tsoumpas C, Mustafovic S, Beisel T, Aguiar P, Dikaios N and Jacobson M W 2012 STIR: software for tomographic image reconstruction release 2 Phys. Med. Biol. 57 867-83

Tofts P S et al 1999 Estimating kinetic parameters from dynamic contrast-enhanced T(1)-weighted MRI of a diffusable tracer: standardized quantities and symbols J. Magn. Reson. Imaging $10223-32$

Tofts P S and Kermode A G 1991 Measurement of the blood-brain barrier permeability and leakage space using dynamic MR imaging: I. Fundamental concepts Magn. Reson. Med. 17357-67

Tsao J, Boesiger P and Pruessmann K P 2003 k-t BLAST and k-t SENSE: dynamic MRI with high frame rate exploiting spatiotemporal correlations Magn. Reson. Med. 50 1031-42

Tsoumpas C, Aguiar P, Nikita K S, Ros D and Thielemans K 2004 Evaluation of single scatter simulation algorithm implemented in the STIR library IEEE Symp. Conf. Record Nucl. Sci. 63361-5

Würslin C, Schmidt H, Martirosian P, Brendle C, Boss A, Schwenzer N F and Stegger L 2013 Respiratory motion correction in oncologic PET using T1-weighted MR imaging on a simultaneous whole-body PET/MR system J. Nucl. Med. 54 464-71

Zheng X, Xiao L, Fan X, Huang N, Su Z and Xu X 2015 Free breathing DCE-MRI with motion correction and its values for benign and malignant liver tumor differentiation Radiol. Infect. Dis. 265-71 